\title{
The influence of the layered character of snow cover on the triggering of slab avalanches
}

\author{
JÜRG SCHWEIZER \\ Eidgenössisches Institut für Schnee- und Lawinenforschung, CH-7260 Weissfluhjoch/Davos, Switzerland
}

\begin{abstract}
One of the principal aims of avalanche warning is to prevent slab avalanches triggered by skiers. Other than explosives, the best practical tool for stability evaluation is the Rutschblock test. Whether the slab may be triggered by a skier depends on various slab characteristics. Important factors seem to be depth of the weak layer, slab hardness and sublayering of the slab. The stress distribution induced by a skier is calculated by the finite element method for typical snow-cover configurations. The additional shear stress is of the same order of magnitude as the shear strength of weak layers. Besides the critical weak layer - prerequisite for a slab avalanche - hard layers seem to be important. The analysis suggests that a shear failure is most probable at the transition from a hard to a soft layer. This corresponds well to observations of slab-avalanche profiles. The results may help to improve and quantify the analysis of snow profiles.
\end{abstract}

\section{INTRODUCTION}

In the Swiss Alps most avalanche victims are skiers, most of whom have probably released the fatal avalanche themselves. The skier as a trigger of slab avalanches is reality. One of the principal aims of avalanche warning is, therefore, to prevent slab avalanches triggered by skiers (Schweizer, 1991).

Sometimes, during and just after snow storms, the avalanche risk is obvious. But what happens afterwards?

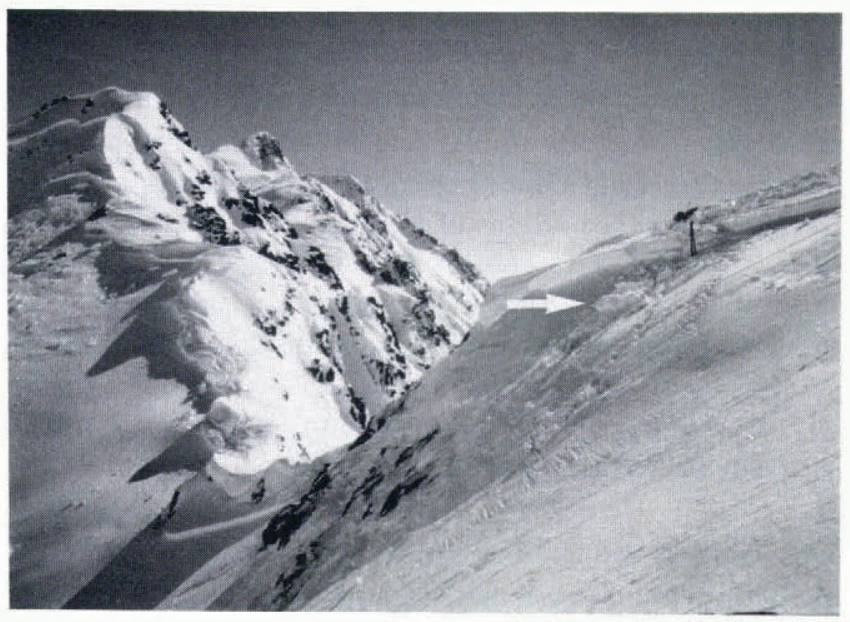

Fig. 1. A slab avalanche at Alpetlilücke (Swiss Alps) triggered by a group of skiers. They made a Rutschblock test near the top of the slope (white arrow). The block could not be released. Minutes later the slope failed. Several were caught and two men were killed (photo by $\mathrm{Hj}$. Etter, EISLF).
As soon as natural avalanches and signs of danger can no longer be observed, the critical phase of risk assessment begins. Will one or several skiers be able to trigger a slab? On which slope and at what time? These are the crucial questions for avalanche forecasters, ski patrollers and backcountry skiers.

Stability tests are a practical, although sometimes dangerous, way to answer the question; explosives are most efficient; snow profiles, shovel and Rutschblock tests are frequently used (Föhn, 1987). All these tests suffer from one problem: areal representativity. It is usually quite difficult to find a test place which is both safe and representative; it is a contradiction. Accidents are known to have occurred after the skiers made a Rutschblock test which indicated a minimal risk only. However, the test site at the top of the slope was badly chosen (Fig. 1). A further critical point is the extrapolation of risk from a test result to a whole region. The variability of the snow cover limits the use of stability tests.

Despite these drawbacks, Rutschblock tests are frequently used in Switzerland to evaluate avalanche risk. The Rutschblock test shows clearly the presence of a critical weak layer, a prerequisite of a slab avalanche (McClung, 1987; Bader and Salm, 1990). Whether a slab can be triggered by a skier depends on the slab characteristics, especially on the sort of snow-cover layering. Snow-cover parameters typically change on different slopes. Hence it is important to emphasize that the result of a Rutschblock test should never directly be connected to a certain degree of danger. The type of release and other snow-cover characteristics must be considered. The problem is clearly shown by two tests made on the same day in the winter of 1991-92. After a snowstorm ( $94 \mathrm{~cm}$ of new snow) on a westerly slope, snow 

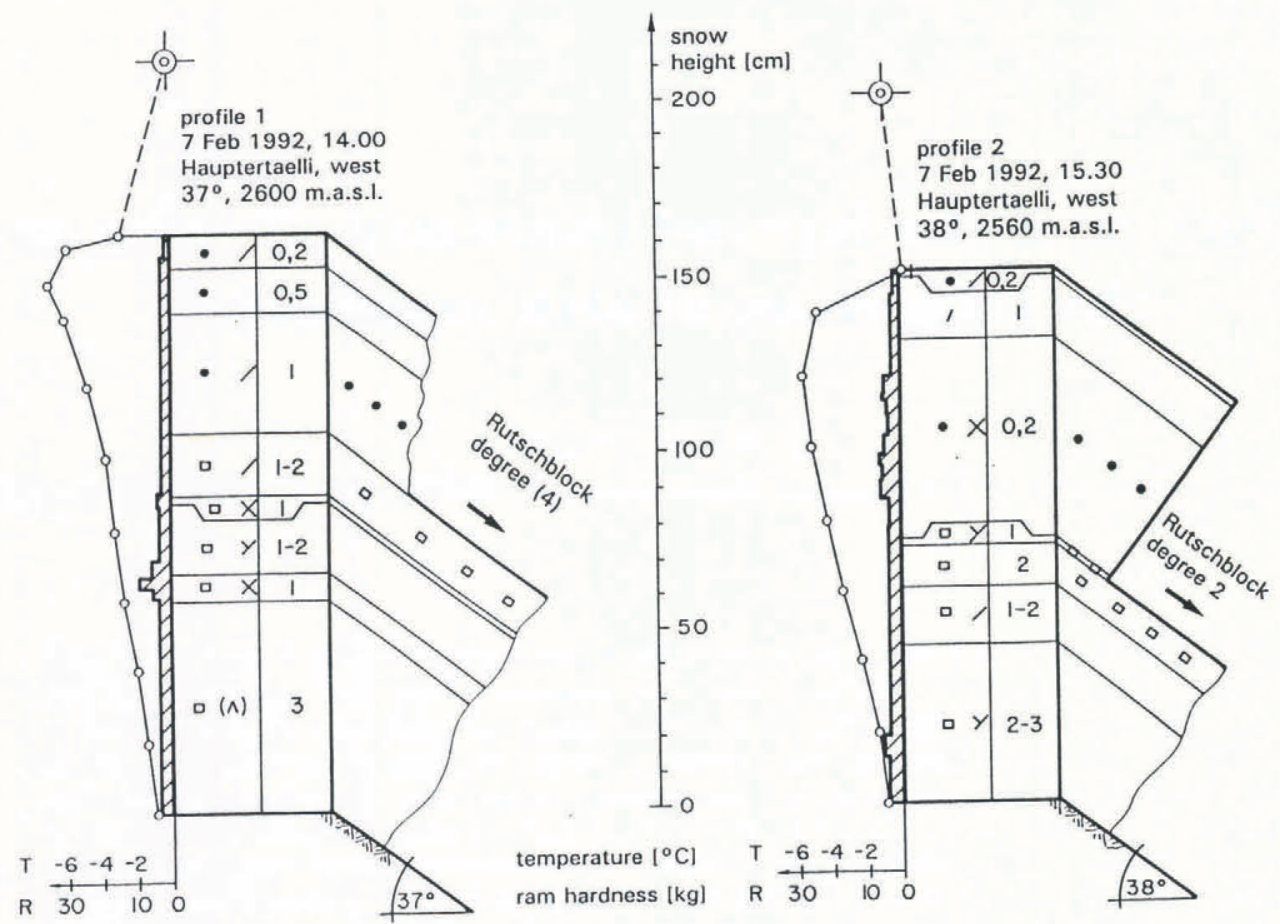

Fig. 2. Two snow profiles with the Rutschblock result. Test sites were on a westerly slope in the Parsenn area (Grisons, Swiss Alps) about $100 \mathrm{~m}$ apart.

profiles with Rutschblock tests were made of two test sites about $100 \mathrm{~m}$ apart (Fig. 2). Although located on the same slope the test results were quite different. At the first site (profile 1) the new snow was rather loose while at the second test site (profile 2) the new snow was more consolidated. This difference on the same slope may have been caused by different wind conditions during the snowstorm due to microrelief. Accordingly, at the second test site the block slipped off easily (degree 2) whereas the first block collapsed with the first jump (degree 4). The slab characteristics seem to be decisive. It is supposed that analysis from a mechanical point of view might give insight into the conditions which are favorable for the release by a skier. Colbeck (1991) pointed out the importance of layering; Curtis and Smith (1974) calculated by the finite element method the stress distribution in a multi-layered snow cover; and Föhn (1987) showed the important effect of the skier and included him in the stability index. The present study will combine the above and try to determine the stress distribution induced by a skier in a typically layered snow cover.

\section{SOME MECHANICAL POINTS}

Snow can be considered as a visco-elastic fluid (Salm, 1982). However, it is difficult to describe snow properly with adequate material properties because knowledge of snow is insufficient. According to the problem, snow is usually considered as either a linear elastic or a linear viscous body. The time scale of the load is decisive. Since stresses and strain rates in this study are most important, it does not matter what is chosen.

Whether a snow mass on a slope is stable depends on stress and strength: if the stress exceeds the strength, the snow slope fails. This view is too simple, in particular since the strength depends on the strain rate induced. Bader and Salm (1990) developed new ideas on the slab release mechanism based on the existence of a super-weak zone. They clearly distinguish between the ductile and the brittle phase of failure. The release of a natural avalanche can be reasonably explained. In this paper it is assumed that a skier (especially a moving one) produces stress peaks and strain rates which are sufficiently large to trigger a brittle failure directly. The initial failure may be a shear failure in the weak layer, a shear failure at the interface of two layers, or the frequently observed collapse of the weak layer ("whumm" phenomenon) leading to the shear failure. The strain rates may be decisive but the details of fracture mechanics are not debated here; this study concentrates on stress calculations. An index of the shear strength of weak layers may be deduced from shearframe measurements. Since the shear frame is usually pulled rather fast, it is assumed that the observed shearstrength values could be of the order of magnitude of brittle strength. Attenuation by soft layers and dynamic loading by a jumping skier are additional practical problems. These effects will be included in a future study.

\section{MODEL CALGULATIONS}

Snow will be considered as a linear viscous body of constant temperature. Using a Cartesian coordinate system with $x-y$ plane parallel to the slope, the following set of equations will describe the snow:

$$
\begin{aligned}
\sigma_{i j, j}+\rho f_{i} & =0, \\
\sigma_{i j}{ }^{\prime} & =\eta \dot{\varepsilon}_{i j}{ }^{\prime}, \\
\sigma_{k k} & =\eta[(m+1) /(m-2)] \dot{\varepsilon}_{k k},
\end{aligned}
$$


where $\sigma_{i j}{ }^{\prime}$ is the stress deviator defined as $\sigma_{i j}-\frac{1}{3} \delta_{i j} \sigma_{k k}, \sigma_{i j}$ is the stress tensor, $\rho$ is the density, $f_{i}$ is the specific body force, $\eta$ is viscosity, $\dot{\varepsilon}_{i j}$ is strain-rate tensor defined as $\frac{1}{2}\left[u_{i, j}+u_{j, i}\right], m$ is the inverse of the viscous analogue of Poisson's ratio, $u_{i}$ is velocity, $\delta_{i j}$ is Kronecker symbol, and ,$j$ is the partial derivative with respect to $x_{j}$. In all equations Einstein's summation convention is used. Equation (1) is the balance of momentum or the equilibrium equation (neglecting accelerations) and Equations (2) and (3) are constitutive relations describing the linear viscous compressible behavior (Mellor, 1975).

To determine the force a skier exerts on the snow cover, a line load on a semi-infinite half space can be considered. This problem has a well known analytical solution first derived by Boussinesq (Salm, 1977; Föhn, 1987). The line load is assumed to be $500 \mathrm{~N} \mathrm{~m}^{-1}$.

A two-dimensional vertical section of the snow cover parallel to the slope angle is shown in Figure 3. Slope angle is $30^{\circ}$, snow thickness is $1 \mathrm{~m}$ and the modelled section is $10 \mathrm{~m}$ long. Boundary conditions are a stress-free surface (except the line load), no motion at the bottom (frozen to the ground), and corresponding forces at the front and the back side to balance the snow block.

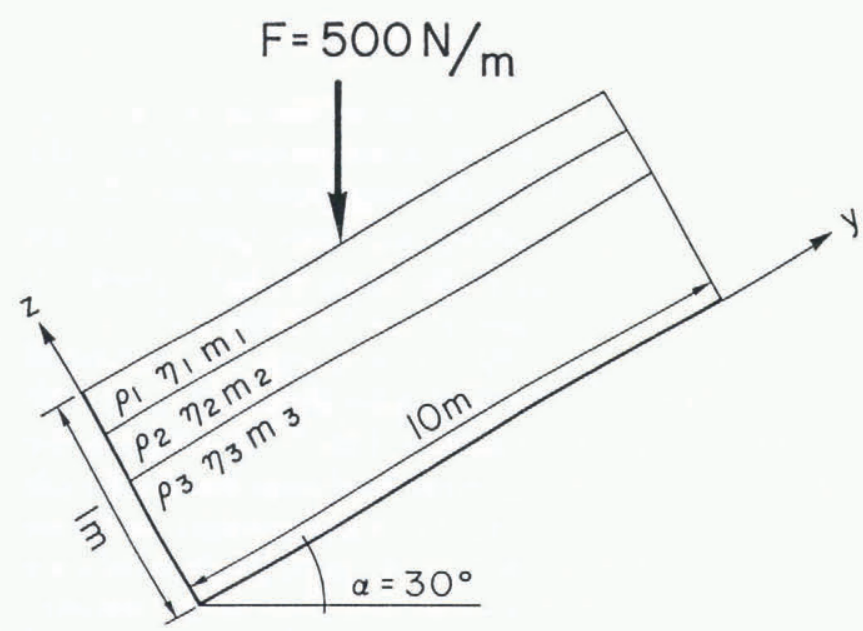

Fig. 3. Geometry of the modelled section of the layered snow cover.

Typical ram or hardness profiles were modelled to study the effect of the layered character of the snow cover. Since hardness is not a physical parameter, it is assumed that the hardness can partly be described by the viscosity. A hard layer is characterized by a high viscosity and a soft layer by a low viscosity. The hardness profiles were simplified to consist of only three layers: so-called soft, medium and hard layers. Values of the density and of $m$ were chosen arbitrarily (Table 1) to determine the viscosity by a relationship derived by Claus (1978):

$$
\eta=\frac{1}{2}[(m-2) /(m-1)] C \exp \{\alpha \rho\} \exp \{E / R T\},
$$

where $C$ is $1.8182 \times 10^{-6} \mathrm{~Pa} \mathrm{~s}, \alpha$ is $0.0207 \mathrm{~m}^{3} \mathrm{~kg}^{-1}, E$ is $67.3 \mathrm{~kJ} \mathrm{~mol}^{-1}$ and $R$ is $0.0083 \mathrm{~kJ} \mathrm{~mol}^{-1} \mathrm{~K}^{-1}$. The temperature, $T$, is assumed to be $268 \mathrm{~K}$.

Figure 4 gives the simplified hardness profiles which were studied. Profile A consists of a hard layer of $40 \mathrm{~cm}$ above a soft ground layer. Profile B is the contrary, i.e.
Table 1: Material properties of typical snow layers used

\begin{tabular}{lccc}
$\begin{array}{c}\text { Layer } \\
\text { characteristic }\end{array}$ & Density $\rho$ & Viscosity $\eta$ & Number \\
& $\mathrm{kg} \mathrm{m}^{-3}$ & Pa s & \\
& & & \\
\hline Soft & 100 & $8.9 \times 10^{7}$ & 8 \\
Medium & 200 & $5.7 \times 10^{8}$ & 4 \\
Hard & 300 & $2.4 \times 10^{9}$ & 2.5
\end{tabular}

soft snow on a hard layer. Profiles C and D are three-layer cases: soft-hard-medium and hard-soft-medium, respectively. Profile E is similar to profile B and is used for comparison. According to de Quervain and Meister (1987) the profiles A, C and D are probably more dangerous than profiles $\mathrm{B}$ and $\mathrm{E}$. The very weak thin layer (also called Gleitschicht) is usually not modelled, but is assumed to be about $60 \mathrm{~cm}$ above the ground. The $40 \mathrm{~cm}$ above the weak layer is the slab, and the $60 \mathrm{~cm}$ below is the ground layer.

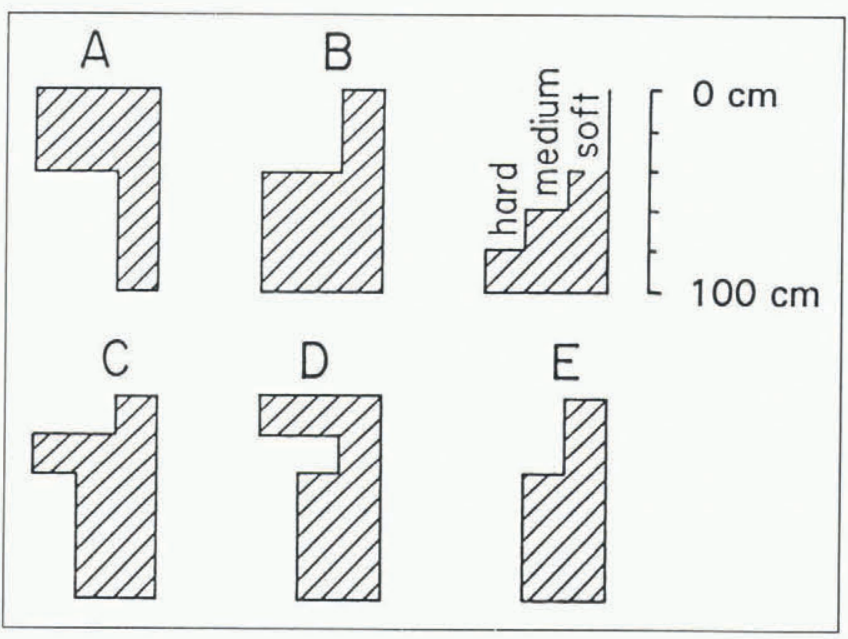

Fig. 4. Simplified hardness profiles. The profiles consist of three layers of soft, medium or hard snow.

To accommodate more complex geometries the problem will be solved numerically by the finite element method. The FEM-program TPS10 was used. The meshes contained up to 2121 nodes, 2000 four-node elements, and were quite fine to the top and to the middle where the line load causes the largest stresses. All numerical calculations were performed on a 32-bit Hewlett-Packard HP 330 computer.

\section{RESULTS}

The analytical solutions of laminar flow (creep) and for stress distribution due to a static line load were used to test the numerical solution by the finite element method. 


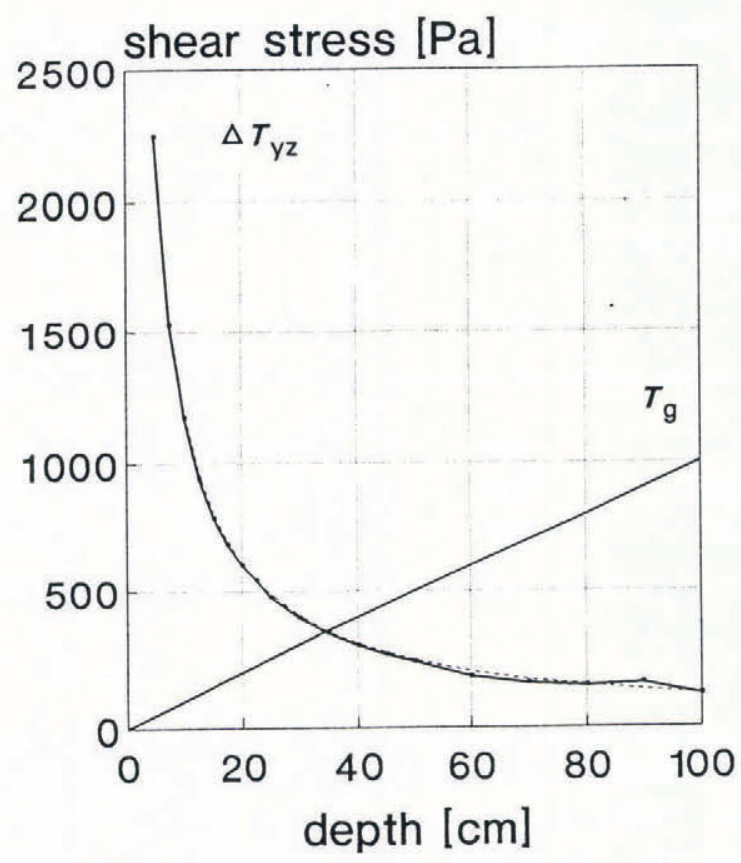

Fig. 5. Numerically calculated maximal additional shear stress $\left(\Delta \tau_{y z}\right)$ a skier exerts on an idealized snow cover vs depth, z. Shear stress due to gravity is also shown. Dashed line gives the analytical solution.

Figure 5 shows the maximal additional shear stress, $\Delta \tau_{y z}$, that a skier exerts on a homogenous, incompressible snow cover $\left(\rho=200 \mathrm{~kg} \mathrm{~m}^{-3}, \eta=7.80 \times 10^{7} \mathrm{~Pa} \mathrm{~s}, m=2.1\right)$. The numerical solution is compared to the analytical one. The numerical solution continously converges to the analytical one, if the grid is refined. The influence of the skier is most pronounced near the surface. At a depth of about $35 \mathrm{~cm} \Delta \tau_{y z}$ is about as large as the shear stress, $\tau_{g}$, due to the weight of the overlying snow column.

All stresses $-\sigma_{y}, \sigma_{z}$ and $\Delta \tau_{y z}$ - are shown along a surface parallel to the slope for two depths in Figure 6. The snow cover is assumed to be homogenous. Physical parameters are equal to the ones of the so-called hard layer (Table 1). The areal effect of the skier is visible for
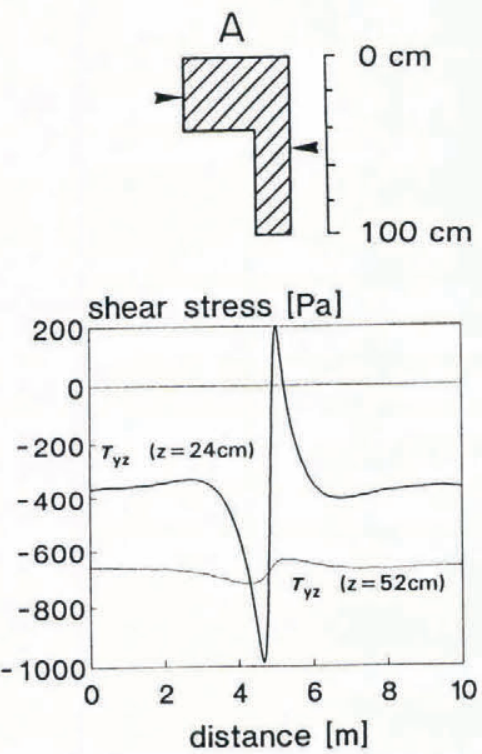

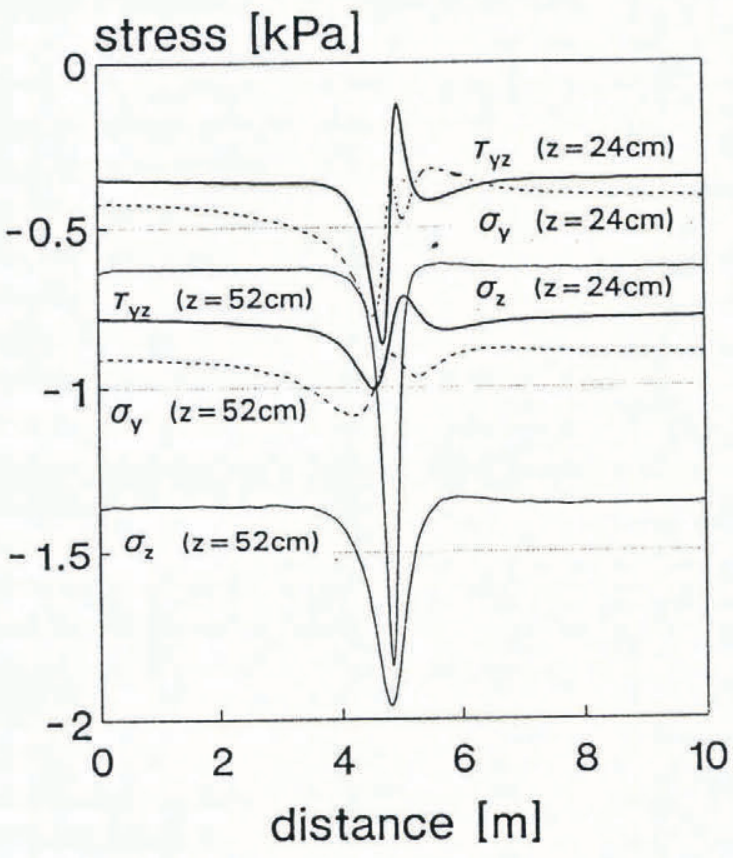

Fig. 6. Total stresses $\left(\sigma_{y}, \sigma_{z}\right.$ and $\left.\Delta \tau_{y z}\right)$ in a homogenous snow cover including the effect of a skier at depths of 24 and $52 \mathrm{~cm}$.

about $1 \mathrm{~m}$ around his position in the layer near the surface. The stress curves are more pronounced near the surface. At greater depth the magnitude of the effect of the skier is obviously smaller but more spread out; this follows from the image of the pressure bulb. (Figure 6 partially reproduces a similar one by Salm (1977).)

The idealized two-layer snow covers are considered next (profiles $\mathrm{A}$ and $\mathrm{B}$ ). The shear stress, $\Delta \tau_{y z}$, for both profiles is shown in two depths (Fig. 7). In profile A the effect of the skier is larger in the hard layer than in the soft one of profile B, whereas in profile B the stresses are better transferred to depth. The hard layer in profile A forms a sort of bridge which takes up most of the skier's impact.

Maximal additional shear stresses, $\Delta \tau_{y z}\left(=\tau_{y z}-\tau_{g}\right)$, of
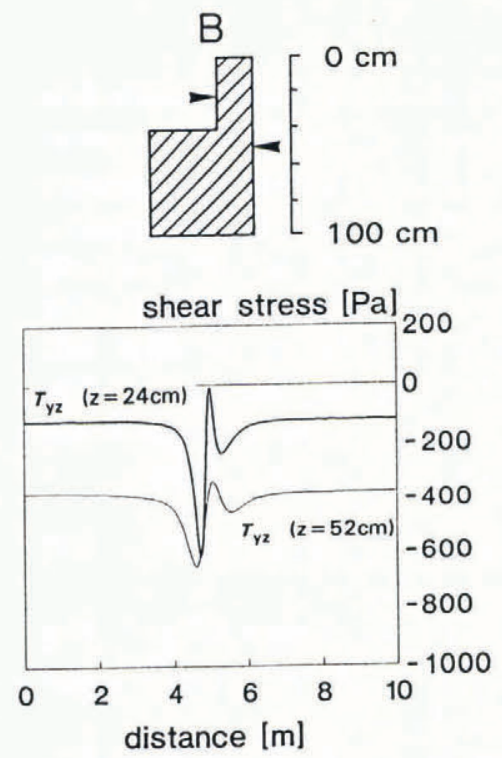

Fig. 7. Shear stress, $\tau_{y z}$, along the modelled section at depths of 24 and $52 \mathrm{~cm}$ are given for the layered snow cover described by profile $A$ (left) and profile $B$ (right). 


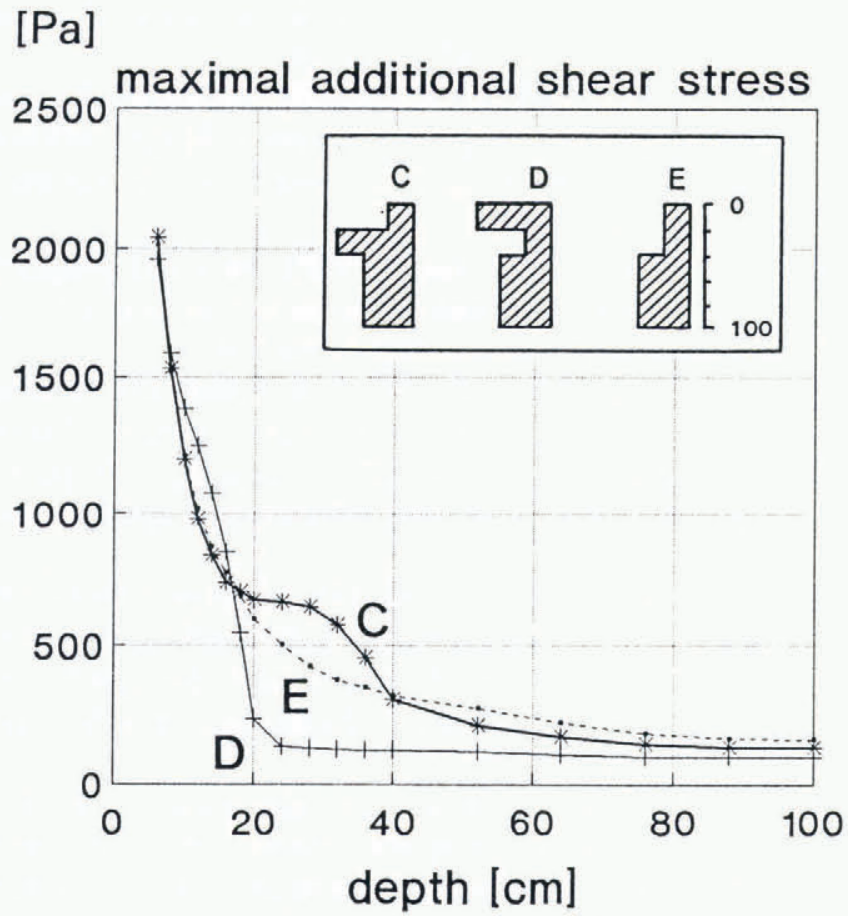

Fig. 8. Maximal additional shear stress, $\Delta \tau_{y z}$, a skier exerts on the layered snow cover described by profiles $C, D$ and $E$ vs depth, $z$.

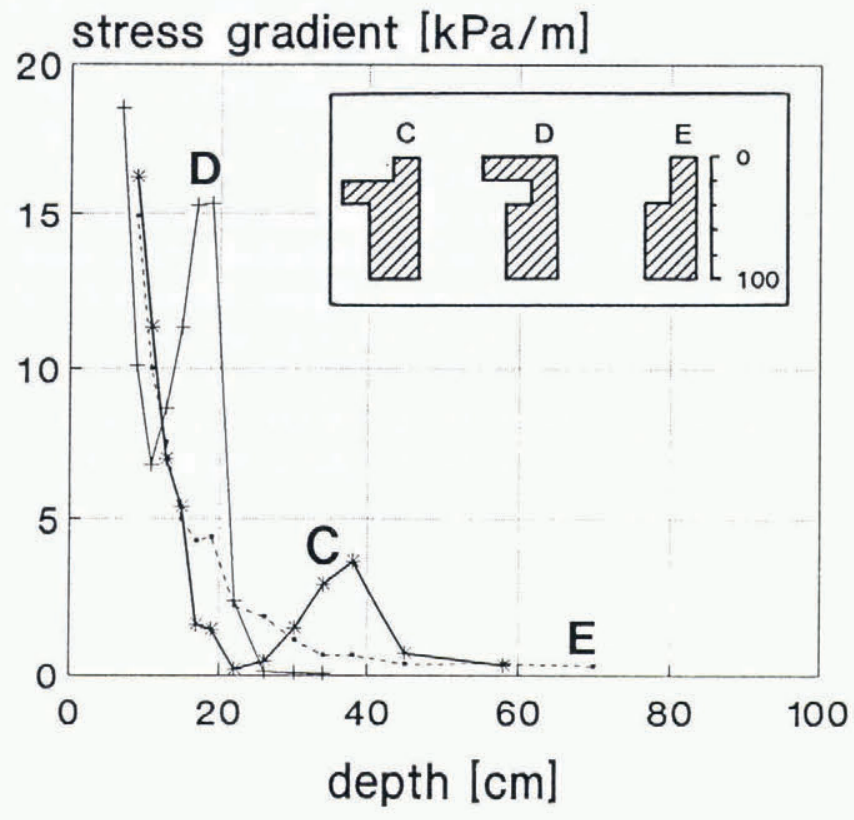

Fig. 9. Spatial gradient of maximal additional shear stress $\left(\Delta\left(\tau_{y z}\right) / \Delta z\right)$ vs depth, $z$, for the layered snow cover described by profiles $C, D$ and $E$.

the three-layer profiles $\mathrm{C}$ and $\mathrm{D}$ and of profile $\mathrm{E}$ are given in Figure 8. The layer configuration influences the additional shear stress at a given depth, e.g. at $z=$ $30 \mathrm{~cm}$ the differences between the different models are quite clear: $610 \mathrm{~Pa}$ in profile $\mathrm{C}, 400 \mathrm{~Pa}$ in profile $\mathrm{E}$ and only $130 \mathrm{~Pa}$ in profile $\mathrm{D}$. These results correspond to the ones given in Figure 7. No stress peaks could be found at the transition from one layer to another. The maximal additional shear stress decreases continously, but not
$[\mathrm{Pa}]$

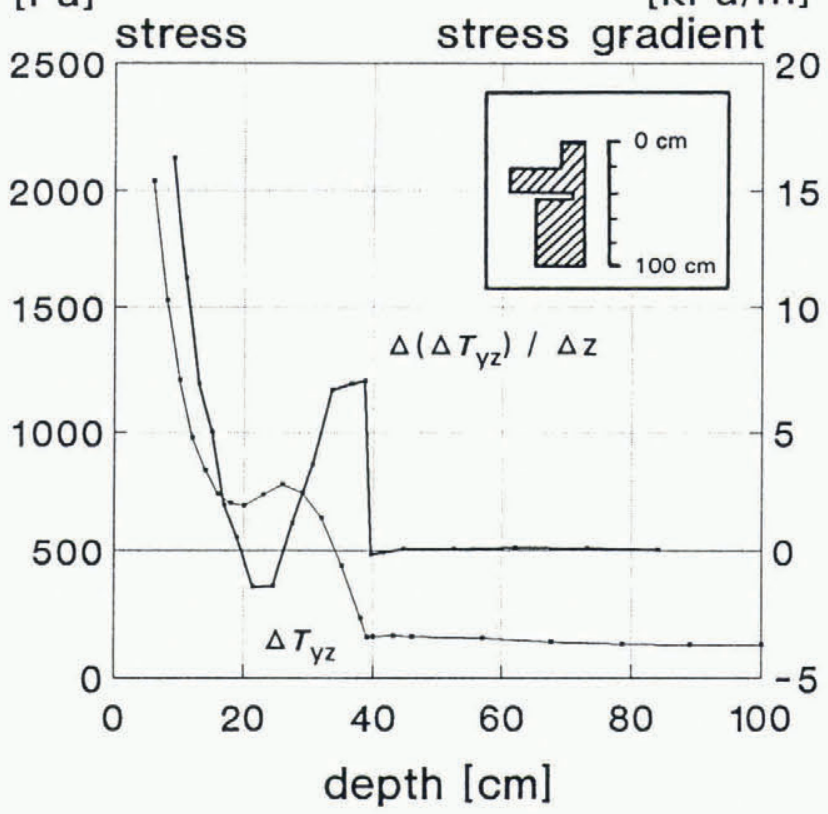

Fig. 10. Maximal additional shear stress $\left(\Delta \tau_{y z}\right)$ and spatial gradient of maximal additional shear stress $\left(\Delta\left(\Delta \tau_{y z}\right) / \Delta z\right)$ vs depth, $z$, for the layered snow cover with a very soft layer beneath the hard layer (similar to profile $C$ ).

uniformly as in the case of a homogenous snow cover. Bends occur at the depth of the transition from one layer to another. These discontinuities are decisive. There is a sudden change in the spatial gradient of the shear stress which can be shown by differentiating the stress curves of Figure 8. This was done in a simplified manner by determining the stress difference between adjacent data points divided by the difference in depth: $\Delta\left(\Delta \tau_{y z}\right) / \Delta z$ (Fig. 9). The curves of stress gradient do not decrease continously. Secondary maxima occur at the depths of layer transition, more precisely at the transition from a hard layer to a soft one. There the peak of the stress gradient is larger and sharper for the snow cover described by profile $\mathrm{D}$ than for the one decribed by profile E. This follows from the smaller depth of the transition from the hard to the soft layer.

Finally, a very soft layer of $2 \mathrm{~cm}$ thickness was introduced just below the hard layer of profile C. The physical parameters of the very soft layer were $\rho=$ $50 \mathrm{~kg} \mathrm{~m}^{-3}, \eta=3.1 \times 10^{7} \mathrm{Pas}$, and $m=10$. The peak of the stress gradient at the transition of the hard to the very soft layer $(z=40 \mathrm{~cm})$ is about twice as large as in profile C (Fig. 10). The analysis of the strain rates vs depth shows a maximum of the strain rates in the very soft layer. That means most of the deformation of the snow cover occurs within the weak layer. The larger the difference of viscosity of two adjacent snow layers, the more pronounced the peak of stress gradient seems to be.

\section{CONCLUSIONS AND OUTLOOK}

The additional calculated shear stress produced by a skier in a near-surface layer is of the same order of magnitude as the shear strength of weak layers (Mellor, 1975). The 
layering of the snow cover has a significant influence on the stress distribution induced by a skier. The additional stress is most pronounced in hard layers described by high viscosity values. Thick hard layers form a sort of bridge. Even if there are weak layers deeper down, the slope will be stable. For instance a depth-hoar layer is not dangerous if there is a thick consolidated layer above, as long as the snow cover does not become wet.

The occurrence of a peak in the stress gradient at the transition from a hard to a soft layer is remarkable. The peak corresponds to the sudden transition from small to large deformations. The snow must be stressed additionally near the interface. Consequently a failure seems to be most probable just beneath a hard layer, at the transition to softer, more deformable snow. The larger the difference in the viscosity the more dangerous the slope seems to be, and the shallower the hard layer the more probable is a failure. These results correspond well to observed profiles from slab avalanches released by skiers. A shear failure just beneath a hard layer is typical.

We will try to quantify the above rules by further modelling. The aim is to introduce the results in an expert system which will analyse the stability of snow profiles. Field experiments are planned to measure the skiers' impact. It seems that most of the static load is damped off but dynamic loading (jumps) might overcome the damping. To be able to model what will be measured, the constitutive relations will probably have to be improved substantially.

\section{ACKNOWLEDGEMENT}

Thanks are due to P. Föhn for guidance and for many stimulating discussions.

\section{REFERENCES}

Bader, H. P. and B. Salm. 1990. On the mechanism of snow slab release. Cold Reg. Sci. Technol., 17(3), 287300 .
Colbeck, S. C. 1991. The layered character of snow covers. Rev. Geophys., 29(1), 81-96.

Claus, B. Unpublished. Compactive viscosity of snow from settlement gauge experiments. Eidgenössisches Institut für Schnee- und Lawinenforschung. Interner Bericht 565, 1978.

Curtis J. O. and F.W. Smith. 1974. Material property and boundary condition effects on stresses in avalanche snow-packs. 7. Glaciol., 13(67), 99-108.

Föhn, P.M.B. 1987. The stability index and various triggering mechanisms. International Association of Hydrological Sciences Publication 162 (Symposium at Davos 1986 - Avalanche Formation, Movement and Effects), 195214.

McClung, D. M. 1987. Mechanics of snow slab failure from a geotechnical perspective. International Association of Hydrological Sciences Publication 162 (Symposium at Davos 1986 - Avalanche Formation, Movement and Effects), 475-508.

Mellor, M. 1975. A review of basic snow mechanics. International Association of Hydrological Sciences Publication 114 (Symposium at Grindelwald 1974 - Snow Mechanics), 251-291.

Quervain, M.de and R. Meister. 1987. 50 years of snow profiles on the Weissfluhjoch and relations to the surrounding avalanche activity (1936/37-1985/86). International Association of Hydrological Sciences Publication 162 (Symposium at Davos 1986 - Avalanche Formation, Movement and Effects), 161-181.

Salm, B. 1977. Snow forces. J. Glaciol., 19(81), 67-100.

Salm, B. 1982. Mechanical properties of snow. Rev. Geophys. Space Phys., 20(1), 1-19.

Schweizer, J. 1991. Dry slab avalanches triggered by skiers. In Proceedings of the International Snow Science Workshop, October 9-13, 1990, Bigfork, Montana U.S.A. Bigfork, MT, ISSW'90 Committee, 307-309.

The accuracy of references in the text and in this list is the responsibility of the authors, to whom queries should be addressed. 